\title{
EL CULTIVO “IN VITRO" COMO INSTRUMENTO PRÁCTICO PARA EL DIAGNÓSTICO Y AISLAMIENTO PRIMARIO DE LEISHMANIA BRAZILIENSIS BRAZILIENSIS. 2. Estudios en pacientes de áreas endémicas *
}

C. A. Giba Guba, E. M. NETTO, . L. M. COSTA, A. C. BaRRETO \& P. D. MARSDen

\section{R E S U M E N}

El cultivo "in vitro" de Leishmania braziliensis braziliensis constituye un método útil en el trabajo de campo, para el aislamiento primario de ésta subespécie de Leishmania. Cultivos en dos medios difásicos de agar sangre (DAB y EVANS) y dos medios líquidos (SCHNEIDER'S y AR-103) realizados en pacientes con lesiones cutáneas de Leishmaniasis Tegumentaria Americana (LTA) demostraron: 1) Similar sensibilidad de los medios DAB y Schneider's cuando utilizamos el proceđimiento de aspiración de las muestras con aguja. 2) Rendimien. to sensible y reproducible, con el medio DAB, cuando comparado, en repetidas ocasiones, con el medio EVANS. 3) Incremento significativo en el aislamiento primario de Leishmania braziliensis brazilensis mediante la ejecución, en la misma lesión de cada paciente, de tres aspiraciones consecutivas en sitios diferentes de la úlcera activa ( $50 \%$ de positividad, con el medio DAB).

UNITERMOS: Leishmaniose tegumentar - Leishmania braziliensis braziliensis - Diagnóstico pelo cultivo in vitro.

\section{N T R O D U G G I O N}

El aislamiento del parásito, en Leishmaniasis Tegumentaria Americana (LTA), es todavia necesario para el diagnóstico y prognóstico de la enfermedad 17. Las nuevas técnicas para la identificación "in situ" de los amastigotes de Leishmania usando anticuerpos monoclonales e hibridización del k-DNA 23.2 no son todavia apli. cables en la investigación epidemiológica de campo.

Un método de aislamiento de Leishmania braziliensis braziliensis insuficientemente explorado, en investigaciones de terreno, es el del cultivo "in vitro" de los parásitos de infecciones humanas 4,19. Observaciones evaluando la sensibilidad de los medios de cultivo recomendados $^{9}$ solamente son posibles de ser realizadas en áreas de reconocida endemicidad de la
L. b. braziliensis, subespecie de difícil aislamiento y cultivo $11,12,13$.

Este trabajo presenta nuestros progressos alcanzados a lo largo de los ûltimos 3 años, en el aislamiento de $\mathbf{L}$. b. braziliensis de pacientes con lesiones cutáneas de LTA en una área de estudio longitudinal en Bahia, Brasil 3,6,7. Los resultados obtenidos sugieren que, el método cultural es efectivo, sensible y práctico en los trabajos epidemiológicos de ésta endemia.

\section{MATERIAL Y METODOS}

\section{1) Los pacientes}

Fueron comprendidos en éste estudio, 9 series de pacientes investigados en las regiones

Núcleo de Medicina Tropical e Nutriçăo, Universidade de Brasília, Caixa Postal 153121, 70.910 Brasília, D.F., Brasĭ Trabajo parcialmente financiado por CNPq (Pro. 403682/82) y OMS/TDR-RE (820526) 
CUBA CUBA, C. A.; NETTO, E. M.; COSTA, J. L. M.; BARRETO, A. ᄂ. \& MARSDFN, P. N. - El eultivn ". :il.c" como instrumento práctico para el diagnóstico y aislamiento primario de Leishmania braziliensis braziliensis. 2. Estudios en pacientes de áreas endémicas. Rev. Inst. Med. trop. São Paulo, 28:317-324, 1986.

endémicas de Três Braços, Cravolândia y Corte de Pedra, Valença, Bahia; ésta última localidad proporcionó $96 \%$ de los casos investigados. Todos eran pacientes sin tratamiento previo antileishmanial.

\section{2) Procedimientos en la demonstración de Leishmania}

Para la realización de los cultivos en las lesiones de los pacientes, limpiábamos cuidađo. samente la sixperficie del área a ser trahajada mediante la utilización en forma secuencial, de los sigtuientes elenientos: agua oxigenada, agua destilada esteril con detergente, alcohol yodado y finalmente alcohol de $95 \%$. Posteriormente, procedíase a anestesiar por la infiltración de Lidocaine $2 \%$.

La aspiración del material de las lesiones fué hecha por dos procedimientos: 1) Por la utilización de pipeta Pasteur, de punta afilada, unida a una pequeña bombilla de jebe y, 2) Por el uso de una jeringa de $5 \mathrm{ml}$ y aguja de $22 \mathrm{~g}$, conteniendo $0.304 \mathrm{de} \mathrm{ml}$ de solución fisiolo gica esteril. La aspiración, en ambos procedimientos. siguió las recomendaciones de HENDRICKS \& WRIGHT 9 .

En un primer grupo de 33 pacientes ambos procedimientos descritos fueron empleados comparativamente. Posteriormente $\mathrm{y}$ en vista de la ventaja práctica de agujas y jeringas descartables, solamente, la aspiración con éste ma. terial fue empleada.

Paralelamente fueron realizados frotices ("improntas") confeccionados con material de biopsia ("punch", $4 \mathrm{~mm}$ ), o por raspado del margen interno de las lesiones con palito de fósforos ${ }^{20}$, cuyos resultados no son presentados en ésta comunicación por ser tema de otra publicación.

\section{3) Medios de cultivo utilizados}

Los medios empleados en el presente trabajo fueron los mismos descritos en trabajo anterior de ésta seriel6. Citamos el SCHNEIDER'S Drosophila Medium ${ }^{10}$, el DIFCO Blood Agar Medium 21, DAB, el AR.103 medio químicamente definido $1 \mathrm{y}$ el medio de EVANS ${ }^{8}$. Todos los medios contenian Garamicina (Garamycin, Schering, USA, $250 \mathrm{ug} / \mathrm{ml}$ de medio), y cuando indicado, 5-Fluorocytosine (SIGMA, USA, 150 $\mathrm{ug} / \mathrm{ml}$ )

Todos los cultivos fueron incubados a $23^{\circ} \mathrm{C}$ $\pm 1^{\circ} \mathrm{C}$, y examinados diariamente por 7 días consecutivos $\mathrm{y}$, posteriomente, a los 15 y 30 días pos inóculo. Todas las observaciones fue ron hechas en microscopio invertido, como de rutina.

Posteriores estudios de aislamiento primario $\mathrm{y}$ diagnóstico fueron efectuados en la localidad de Corte de Pedra, Bahia, empleando comparativamente el Medio DAB y el EVANS. El procedimiento empleado fué el de la aspiración con aguja y jeringa de muestras de tejidos de las úlceras de pacientes cutáneos. Todos los cultivos positivos fueron transferidos para el medio de propagación de Schneider (complementado con $20 \%$ de suero bovino fe. tal) para la preparación de masa de células para identificación taxonómica.

Finalmente en los últimos estudios, inoculamos 3 Tubos de Medio DAB con muestras de tejidos tomadas de tres diferentes sitios de la misma uilcera de cada paciente ( 2 series de 49 y 15 pacientes con Iesiones cutáneas, respectivamente).

\section{4) Identificación del paràsito}

Los primeros 11 aislados ("stocks") fueron caracterizados e identificados por las téc. nicas de Anticuerpos monoclonales (IFAT $\operatorname{IgG}^{7}$, por el del padrón isoenzimático en acetato de celulosa $\mathrm{y} / \mathrm{o}$ el padrón de desarrollo en Lutzomya whitmani 5 . Posteriormente nosotros encontramos ser más práctico el uso de los anticuerpos monoclonales, utilizando estos "marcadores" específicos y subespecie especificos para Leishmania.

\section{5) Análisis Estadístico}

Las siguientes pruebas estadísticas fueron aplicadas en el análisis de los datos: 1) Test $x^{2}$, ji cuadrada, 2) Test de Cochran, 3) Test " $t$ " Student's. 
CUBA CUBA, C. A.; NETTO, E. M.; COSTA, J. L. M.; BARRETO, A. C. \& MARSDEN, P. D. - El cultivo "in vitro" como instrumento práctico para el diagnóstico y aisiamiento primario de Leishmania braziliensis braziliensis. 2. Estudios en pacientes de áreas endémicas. Rev. Inst. Med. trop. SẼo Paule, \$\$:317-324, 1986.

\section{RESULTADOS}

La TABLA I muestra que utilizando el procedimiento del aspirado, con aguja y jerin. ga, $7(21 \%)$ cepas de $\mathbf{L}$. b. braziliensis fueron aisladas en el medio de Schneider y, $5(15 \%)$ crecieron en el medio de agar sangre DIFCO
(DAB). Esos resultados no son significativa. mente diferentes $(=0.05)$. No obtuvimos diferencia significativa cuando comparamos también ambos procedimientos đe colecta đel ma. terial de las lesiones (Pipeta Pasteur $x$ Aspirado, con aguja y jeringa), en el estudio realizado con el medio DAB.

T A

Aislamiento primario y características de crecimiento de Leishmania. Comparación de tres medios de cultivo, dos procedimientos de colecta de las muestras y tiempo de aparecimiento de los promastigotes "in vitro"

\begin{tabular}{|c|c|c|c|c|c|}
\hline \multirow{3}{*}{$\begin{array}{c}\text { Paciente } \\
\text { n. }\end{array}$} & \multicolumn{4}{|c|}{ Medio de cultivo/Procedimiento de cultivo } & \multirow{3}{*}{$\begin{array}{l}\text { Identificación } \\
\text { taxonómica del } \\
\text { aislado *** }\end{array}$} \\
\hline & \multirow{2}{*}{\multicolumn{2}{|c|}{$\begin{array}{c}\text { D A B* } \\
\text { Aspiración con }\end{array}$}} & \multirow{2}{*}{$\begin{array}{l}\text { Schneider } \\
\text { Aspiración } \\
\text { con aguja }\end{array}$} & \multirow{2}{*}{$\begin{array}{l}\text { A R- } 103 \\
\text { Aspiración } \\
\text { Con aguja }\end{array}$} & \\
\hline & & & & & \\
\hline & & & & & \\
\hline 1 & - & - & - & - & \\
\hline 2 & - & - & - & - & \\
\hline 3 & - & - & - & - & \\
\hline 4 & - & - & - & - & \\
\hline $\mathbf{5}$ & - & - & - & - & \\
\hline 6 & - & - & - & - & \\
\hline 7 & - & - & - & - & \\
\hline 8 & - & $\rightarrow$ & - & - & \\
\hline 9 & - & - & $+(10) * *$ & - & L. b. b. (I, Mabs $)$ \\
\hline 10 & - & - & $+(10)$ & - & L. b. b. (I, MAbs) \\
\hline 11 & - & - & - & - & \\
\hline 12 & - & - & - & - & \\
\hline 13 & $+(2)$ & $+(2)$ & $+(2)$ & - & L. b. b. ( $I$, MAbs) \\
\hline 14 & - & $\rightarrow$ & - & - & \\
\hline 15 & - & - & - & - & \\
\hline 16 & $+(10)$ & - & - & - & L. b. b. (MAbs) \\
\hline 17 & $+(4)$ & - & - & - & L. b. b. (MAbs, S.) \\
\hline 18 & $+(5)$ & $+(15)$ & - & - & d. b. b. (MAbs) \\
\hline 19 & - & - & - & - & \\
\hline 20 & - & $+(15)$ & $+(15)$ & - & L. b. b. (I, MAbs; S) \\
\hline 21 & $+(4)$ & $+(3)$ & $+(10)$ & - & L. b. b. ( $I$, MAbs ) \\
\hline 22 & $+(3)$ & - & - & - & L. b. b. (MAbs) \\
\hline 23 & - & - & - & - & \\
\hline 24 & - & - & - & - & \\
\hline 25 & $+(6)$ & - & - & - & L. D. b. (MAbs) \\
\hline 26 & $+(8)$ & - & - & -. & L. b. b. (MAbs) \\
\hline 27 & - & - & - & - & \\
\hline 28 & - & - & - & - & \\
\hline 29 & - & - & - & - & \\
\hline 30 & 一 & - & - & - & \\
\hline 31 & - & - & $+(29)$ & - & \\
\hline 32 & - & - & - & - & \\
\hline 33 & - & - & $+(23)$ & - & \\
\hline Total (\%) & $8(24.2) \wedge$ & $5(15.3) \Delta$ & $7(21.2) \mathrm{A}$ & $0(0)$ & \\
\hline
\end{tabular}

* DAB $=$ Agar Sangre Difco. WALTON et aI (21); AR-103 Medio quimicamente definiido AZEVEDO \& ROITMAN(1)

* El número entre paréntesis es el tiempo (días) de aparecimento de promastigotes en el respectivo tubo de cultivo

*** L. b. b. = Leishmania braziliensis braziliensis; $I=$ Isoenzimas: ALAT, ASAT, G6PD; MAbs $=$ Anticuerpos Monoclonales: 3F4F6 L. b. b. 4B9D10 L.B.; 3E6B11 L.b. 1F9D8 L.m.amaz., $\mathbf{S}=$ Sandflies, Lu.whitmani

$\Delta$ Proporciones no son significativamente diferentes (Test " $\mathrm{t}$ " $\mathrm{p}=0.05$ )

Por otro lado, el medio AR-103, falló totalmente en el aislamiento primario de Leishma. nia.
En relación al tiempo de aparición de promastigotes en los tubos de cultivo inoculados, los 5 cultivos fueron positivos, en el medio DAB 
CUBA cUBA, C. A.; NETTO, E. M.; COSTA, J. L. M.; BARRETO, A. C. \& MARSDEN, P. D. - El cultivo “in vitro" como instrumento práctico para el diagnóstico y aislamiento primario de Leishmania braziliensis braziliensis. 2 . Estudios en pacientes de áreas endémicas. Rev. Inst. Med. trop. São Paulo, 28:317-324, 1986.

entre el 2: y $150^{\circ}$ día, después de sembrados. En el medio Schneider's los 7 “stocks", se pre. sentaron positivos entre el 2.0 y 29. día. En ésta serie de 33 pacientes obtuvimos aproximadamente $25 \%$ de aislamiento primario de Leishmania en el medio DAB. Cinco de los "stocks" fueron identificados como L. b. braxiliensis, por sus padrones electroforéticos con las enzimas ALAT (E.C.2.6.1.2. Alanino Aminotransferasa), ASAT (E.C.2.6.1.1. Asparta. to Aminotransferasa) y G6PD (E.C.1.1.1.49 Glucosa. 6 Fosfato dehidrogenasa). (TABLA I).

Resultados confirmatorios fueron obtenidos con los anticuerpos monoclonales especie y subespecie específicos atravez de la técnica de Inmunofluorescencia Indirecta (IFAT-MAbs).
El padrón Peripylaria fué comprobado en 2 stocks caracterizados previamente por las técnicas de isoenzimas $\mathrm{y}$ serológicas (stocks, $17 \mathrm{y}$ 20).

La TABLA II presenta los resultados de $\mathbf{5}$ observaciones de campo donde el medio DAB fué comparado con el medio de EVANS, entre Enero de 1983 y Abril de 1984. Hubo gran variación en los resultados entre los diversos grupos. En dos ocasiones poca diferencia pudo ser detectada en la capacidad de ambos medios para aislar L. b. braziliensis. En una oca. sión el međio EVANS fué superior, $\mathrm{y}$ en las dos últimas ocasiones el DAB fué definitivamente superior. Las razones para explicar esas variaciones no son claras.

T A B L A II

Comparación de la seiusibilidad de dos medios de cultivo, en condiciones de campo, en 6 ocasiones diferentes en un total de 149 pacientes con lesiones de Leishmaniasis cutánea

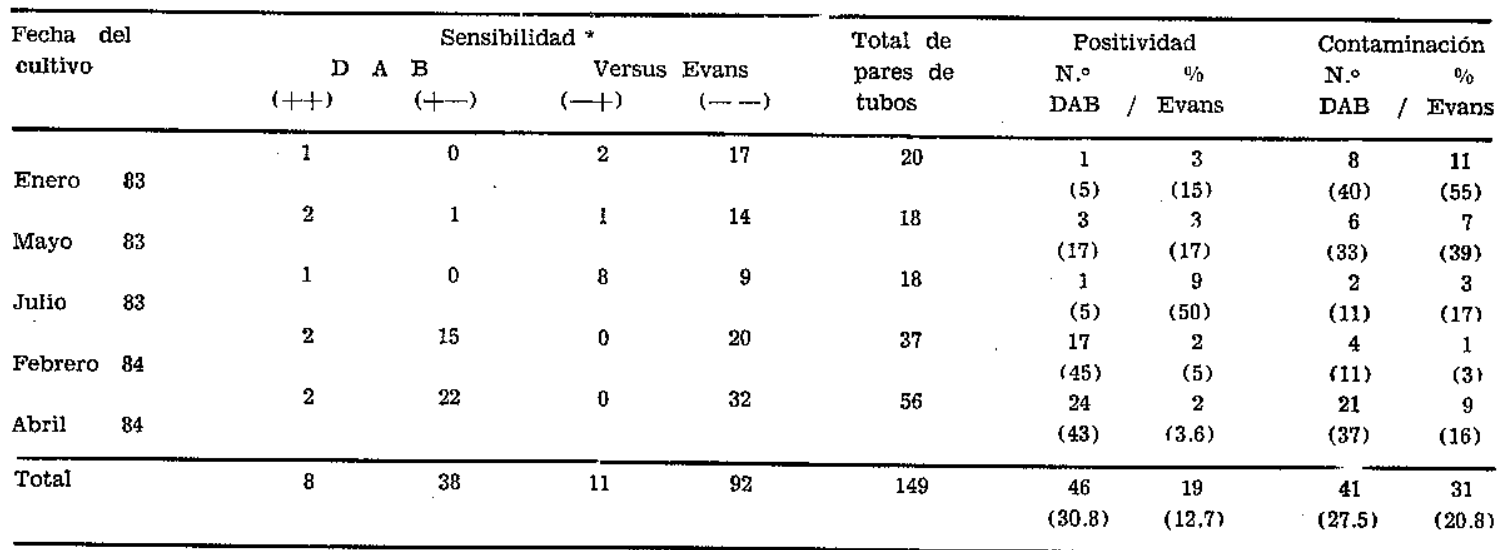

* Los simbolos entre paréntesis indican $=(++)$ positividad por ambos medios; ( + ) positividad por el primer medio, DAB; (-+) positividad por el segundo medio comparado; (- -) negatividad por ambos međios comparados,

Los resultados de las dos últimas observaciones, en 1984, son netamente superiores a Ios 3 estudios previos. La media de aislamien. to para el DAB fué de $30.8 \%$ y para el EVANS, sólo de $12.7 \%$.

La contaminación de los medios, en condiciones de campo, es tolerable, a pesar de la adición de antimicrobianos $(24 \%$ para el DAB y $20 \%$ para los cultivos de EVANS).

En la TABLA III son presentados los resultados obtenidos cuando realizamos, en cada paciente, 3 aspiraciones consecutivas en la misma úlcera leishmanial. Esos resultados demuestran un incremento significativo $(\mathrm{p}<$
0.005 ) en el aislamiento primario de Leishmania. Los porcentajes pasaron de $20.4 \%$, cuando analizamos un solo aspirado, para $51 \%$ después de las 3 aspiraciones inoculadas. individualmente, en un tubo de Medio DAB. Similares resultados fueron obtenidos con otro grupo de 15 pacientes en que conseguimos $26.7 \%$ (en el primer aspirado), 33.3\% (en el segundo) y 46.7 (con el tercer aspirado), respectivamente (no presentados en la TABLA III).

La TABLA IV demuestra que a pesar que las aspiraciones del material de las lesiones fueron ejecutadas en 3 sitios diferentes de la úlcera, no hubo diferencia significativa (Coch 
CUBA CUBA, C. A.; NETTO, E. M.; COSTA, J. L. M.; BARRETO, A. C. \& MARSDEN, P. D. - El cultivo "in vitro" como instrumento práctico para el diagnóstico y aislamiento primario de Leishmania braziliensis braziliensis. 2 . Estuđios en pacientes de áreas endémices. Rev. Inst. Med. trop. São Paulo, 28:317-324, 1986.

T A B I. A III

Aislamiento primário de Leishmania por aspiraciones consecutivas de material de la misma lesión en un grupo de 49 pacientes con lesiones cutáneas

\begin{tabular}{|c|c|c|c|c|c|c|c|}
\hline Aspiraciones & $\begin{array}{l}\text { Positividad/Total i } \\
\text { acumulada }\end{array}$ & de pacientes & $\%$ & Limite & binomial & de & confianzg $95 \%$ \\
\hline 1a. Muestra & 10 & 49 & $20.4^{*}$ & & 10.2 & - & 34.3 \\
\hline 2a. Muestra & 18 & 49 & $36.7^{\star}$ & & 23.4 & - & 51.7 \\
\hline 3a. Muestra & 25 & 49 & $51.0^{\star}$ & & 36.3 & 一 & 65.6 \\
\hline
\end{tabular}

* Diferencia estadística significativa. Test de Cochran, $p<0.005$

$\operatorname{ran} \mathrm{p}>0.1$ ) entre las tres tasas de aislamiento $(10,13 \mathrm{Y} 18$ tubos positivos, respectivamen. te) sugiriendo una uniforme distribución de los amastigotes en la lesión.

T A B L A IV

Interelación entre tres aspiraciones consecutivas em diferentes sitios $y$ en la misma lesion en el aislamiento primario de Leishumania braziliensis braziliensis

\begin{tabular}{|c|c|c|c|c|c|c|c|}
\hline & Primer & Aspirado & Total & & Segundo & Aspirado & Total \\
\hline & + & - & & & + & - & \\
\hline Segundo + & 5 & 8 & $13^{*}$ & Tercer $\quad$ & 9 & 9 & $18^{*}$ \\
\hline Aspirado - & 5 & 31 & 36 & Aspirado - & 4 & 27 & 31 \\
\hline Total & $10^{*}$ & 39 & 49 & Total & 13 & 36 & 49 \\
\hline
\end{tabular}

* Parecen comportarse con la misma sensibilidad pues no existe diferencia estadística significativa, Test de Cochran $p>0.1$

1.० y $20^{\circ}=\mathrm{x}^{2} 0.05<\mathrm{p}<0.1$ (interacción)

$2 .^{\circ}$ y $3 .^{\circ}=\mathrm{X}^{2} 0.001<\mathrm{p}<0.025$ (fuerte interacción)

\section{DISCUSION}

En los estudios epidemiológicos de Leishmaniasis Tegumentaria Americana las técnicas de diagnóstico geralmente visaron la demonstración de los amastigotas de las lesiones. Procedimientos comunmente empleados fueron los frotices, en láminas, histología y la inoculación de hamsteres. Actualmente, aumentó el interés por el aislamiento primario y posterior identificación taxonómica de Leishmania, siendo que los métodos disponibles utilizan los promastigotes del parásito. Hubo entonces la necesidad de mejores $\mathrm{y}$ eficientes medios $\mathrm{y}$ técnicas en el aislamiento primario de Leishma. nia. Se dice que es prioritario identificar los parásitos responsables por las diversas manifestaciones clinicas de la enfermedad 17 especialmente en las infecciones mucocutaneas 18 .

En Ia literatura, han surgido contradicciones relacionadas con la selección del medio más apropiado para el aislamiento primario y mantenimiento "in vitro" de Ieishmania, especialmente de la subespecie $\mathbf{L}$. b. braziliensis $9,10,4$. En la opinión de algunos investigadores como
MAYRINK et al.,15 los cultivos son de poco valor práctico por su contaminación frecuente y porque los parásitos, que fueron aislados "in vitro", también fueron encontrados en los frotices coloreados.

El crecimiento en cultivos de L. b. braziliensis es un problema que ha dificultado, durante mucho tiempo, el progresso de la investigación epidemiológica de leishmaniasis tegumentaria 11,13. Sinembargo, parecería que aún entre los propios aislados de $\mathbf{L}$. b. braziliensis, existe una variacón en su capacidad de adaptación al crecimiento artificial y a su multiplicación en los diversos medios de cultivo. Muy recientemente EVANS et al.18 cultivaron, según describen, en forma fácil $\mathbf{L}$. braziliensis de pacientes que habian contraído la infección en Belize, utilizando los medios difásicos DAB (Blood Agar Difco) y EVANS (Evans Modified, Tobie's Medium). HERRER (comunicación personal) no encuentra dificultades en el cultivo de L. b. peruviana, en $\mathrm{NNN}$ clásico.

Nuestra experiencia, cultivando L. b. braziliensis de Iesiones humanas cutáneas y co- 
CUBA CUBA, C. A.; NETTO, E. M.; COSTA, J. L. M.; BARRETO, A. C. \& MARSDEN, P. D. - El cultivo "in vitro" como instrumento práctico para el diagnóstico y aislamiento primario de Leishmania braziliensis braziliensis. 2. Estudios en pacientes de áreas endémicas. Rev. Inst. Med. trop. São Paulo, 28:317-324, 1986.

lectando el material en los puestos de salud en las áreas endémicas de Bahia demuestra, que L. b. braziliensis es relativamente difícil de aislar y de cultivar (aproximadamente 50\% de éxito en su aislamiento primario). Esto se deduce de nuestros resultados obtenidos con el empleo de los medios DAB y EVANS. En relación al crecimiento en masa de los parásitos, los medios TC-199 y Schneider's, suplementados con $20 \%$ de suero bovino fetal, han demostrado en nuestro laboratorio resultados satisfactorios 16. En seis oportunidades hemos comprobado que aislados de $\mathbf{L}$. b. b. con desarrollo en hamsteres, han fracasado en su crecimiento en medios de cultivo líquidos y de agar sangre (observaciones no publicadas).

Apesar de haber comprobado similar sensibilidad en el aislamiento primario de $\mathbf{L}$. b. b. entre los medios DAB y SCHNEIDER's, concordamos con SHAW \& LAINSON ${ }^{19}$ en señalar que el precio elevado del suero bovino fetal y dificultades en su obtención inviaviliza el empleo del medio líquido monofásico en los estudios de gran escala y de campo, en leishmaniasis.

Posteriormente comparamos la utilidad de los medios difásicos de agar sangre DAB y EVANS, seguramente más económicos $\mathrm{y}$ sensibles ${ }^{16}$. Los resultados se presentaron parcialmente confundidos por un período de aparente anormalidad registrado por el medio de EVANS (Febrero 1983) que inexplicablemente invirtió significativamente su rendimiento. Hemos revisado cuidadosamente la preparación del medio utilizando la propia formulación enviada por el Dr. D. Evans, sin descubrir las razones para dicha variación. El propio EVANS et al., utilizó, con mejores resultados, una mo. dificación del Medio EVANS sin comentar, entretanto; el porque de su abandono y modificación.

Una siguiente etapa Iógica fué la del empleo único del medio DAB de probada sensibilidad en nuestras manos, y perfeccionamiento del procedimiento de colecta del material a inocular. El raciocinio de realizar 3 aspirados consecutivos en diferentes sitios de la misma lesión del paciente mostró que, las aspiraciones previas no interfirieron en la sensibilidad de las subsecuentes, sugeriendo una probable asociación entre la positividad del primero $y$ los dos siguientes aspirados (TABLA 4) y además que los parásitos podrían estar distribuídos uniformemente, en las lesiones activas. Podría ser interesante comparar estos resultados con los obtenidos por el empleo de las 3 aspiraciones inoculadas en el mismo tubo.

Por otro lado, teóricamente, aquellas técnicas que utilizan anticuerpos monoclonales e hibridización del $\mathrm{k}-\mathrm{DNA}$ son las más rápidas $\mathrm{y}$ sensibles para el diagnóstico de pacientes de leishmaniasis, porque ellas pueden ser aplicada directamente en los tejidos o en los frotices de células de las lesiones. Ellas tienen el potencial de poder constituirse, en el futuro, útiles en las investigaciones epidemiológicas de leishmaniasis, sinembargo actualmente son técnicas de laboratório de investigación especializada, WHO ${ }^{23}$. Pensamos que, durante un buen tiempo, continuaremos a usar las técnicas de diagnóstico parasitológico tradicionales que objetivan aislar los promastigotes y amastigotes de Leishmania braziliensis braziliensis. En éste sentido, éste laboratorio, con la metodología de cultivo descrita, es capaz de identificar un aislado de Leishmania, dos semanas después del examen clínico del paciente en la área endémica.

Esto último es obviamente importante para el prognóstico $\mathrm{y}$ tratamiento del indivíduo.

Finalmente, nos encontramos estimulados por haber logrado reducir a $30 \%$ el indice de contaminación de nuestros cultivos. Esta figura es tolerable y quizá compensada por nuestro registro, en condiciones de campo, de $50 \%$ de aislamiento primario del parásito un porcentaje mayor al registrađo por éste laboratorio anteriormente 7 y al descrito para "Uta" $y$ "espundia", por LLANOS CUENTAS et al.14.

\section{SUMMARY}

The viability of the "in vitro" cultivation procedure as a practical tool for diagnosis and primary isolation of Leishmania braziliensis braziliensis. 2. A study of patients from endemic areas.

In vitro cultivation of $\mathbf{L b b}$ is a useful method in the field for primary isolation of 
CUBA CUBA, C. A.; NETTO, E. M.; COSTA, J. I. M.; BARRETO, A. C. \& MARSDEN, P. D. - El cultivo "in vitro" como instrumento préctico para el diagnóstico y alslamiento primario de Leishmania braxiliensis brazillensis. 2. Estudios en pacientes de áreas endémicas. Rev. Inst. Med. trop. Săo Paulo, 28:317-324, 1986.

this leismanial subspecies. Cultures in biphasic blood agar media (DAB and Evans) and liquid media (Schneiders and $A R$ 103) done in patients with cutaneous leismanias showed.

1. A similar sensibility in DAB and Schneiders medium when needle aspirates were used to seed the cultures;

2. That DAB medium performed well when compared with Evans medium;

3. A rise in isolation of $\mathrm{Lbb}$ if 3 aspirations taken from different sites in the same lesion were cultivated $(50 \%$ positivity with DAB medium).

\section{REFERENCES}

1. AZEVEDO, H. P. \& ROITMAN, I. - Cultivation of Trypanosoma cruzi in defined media. In: MOREL, C. M., ed. - Genes and antigens parasites. A laborstory manual. 2nd. ed. Rio de Janeiro, Fundaçāo Oswaldo Cruz, ,1984. p. 29.36.

2. BARKER, D. C. \& BUTCHER, J. - The use of DNA probes in the identification of leishmanias: discrimination between isolates of the Leishmania mexicana and L. braziliensis complex. Trans. roy. Soc. trop. med. Hyg., 77: 285-297, 1983.

3. BARRETO, A. C.; CUBA CUBA, C. A.; MARSDEN, P, D.; VEXANAT, J. \& BELDER, M. - Caracteristicas epidiemiológicas de Leishmaniose Tegumentar Americana em uma regiāo endêmica do Estado da Bahia, Brasil. 1. Leishmaniose humana. Bol. Ofic. sanit. pananer., 90: 415-424, 1981.

4. CHANG, K. P. \& FISH, W. R. - Leishmania In: JENSEN, J. B., ed. - In vitro cultivation protozoa pathogenic to men and domestic animals. Boca Raton, Florida, CRC Press, 1981, p. 2-91.

5. CUBA, C. A.; VEXENAT, A. J.; LLANOS, E. A.; MARSDEN, P. D.; BARRETO, A. C. \& ROSA, A. C. Experimertal infections of wild caught specimens of Lutzomyia (N) whitmani (Diptera, Psychodidae) and their use for Leishmania identification. In: REUNIAOO ANUAL SOBRE PESQUISA BASICA EM DOENCAA DE CHAGAS, 9., Caxambu, 1982. Programa e resumos. Belo Horizonte, CNPq: FINEP, 1982. p. 149.

6. CUBA CUBA, C. A.; NETTO, M. E.: COSTA, L. J.; ROSA, A. de C.; BARRETO, A. C. \& MARSDEN, P. D. - Cutaneous Jeishmaniasis caused by Leishmania braziliensis brazillensis: Improving diagnosis by pr1mary isolation is culture. In: REUNLÄO ANUAL SO. BRE PESQUISA BASICA EM DOENCY DE CHAGAS, 11. Caxambu, 1984. Programa e resumos. Ribeirāo Preto, PIDE: CNPq, 1984. p. 129.

7 CUEA CUBA, C. A.; MrLES. M. A.; VEXENAT, J, A.; MC MAHON PRATT, D.: BARKER, D. C.: BUTCHER, J. D.: BARRETO, A. C. \& MARSDEN, P. D. - A for'st of mucocutaneous leishhmaniasis in Três Braços, Bahia, Brazil. Churacteriwation and identification of Leishmania stocks isolated from man and dogs. Trans. roy. Soc. trop. Med. Hyg., 79: 500-507, 1985.

8. EVANS, D. A.; LANHAM, S. M.; BALDWIN, C. I. \& PETERS, $W$. - The isolation and isoenzyme characterization of Leishmania braziliensis sbsp. from patients with cutaneous leishmaniasis acquired in Betize. Trans. roy. Soc. trop. Meil. Hyg., 78: 35-42, 1984.

9. HENDRICKS, L. D. \& WRIGHT, N. - Diagnosis of cutaneous leishmaniasis by "in vitro" cultivation of saline aspirate in Schneider's Drosophila medium. Amer. J. trop. Med. Hyg., 28: 962-964, 1979.

10. HENDRICKS, L. D. \& CHILDS, G. E. - Present knowledge of the in vitro cultivation of Leishmania. In: THE IN VITRO CULTIVATION OF THE PATHOGENS OF TROPICAL DISEASES. Basel, Schwabe \& Co. AG, 1980. p. 251-272. (Tropical Diseases Research Series No. 3).

11. LAINSON, R. \& SHAW, J. J. - Some problems in studies on parasites of the leishmania braziliensis complex. In: Ecologie des Leishmanioses. Colloquies Internationaux du CNRS, n.० 239. Montpellier, 1977. p. 83-86.

12. LAINSON, R. \& SHAW, J. J. - The role of animals in the epidemiology of South American Leishmaniasis. In: LUMSDEN, W. H. R. \& EVANS, D. A., ed. Biology of the kinetoplastida. London, Academic Press. 1979. v. 2, p. 1-116.

L3. LAINSON, R. - OUr present knowledge of the eco. logy and control of Leishmaniasis in the Amazon region of Brazil. Rev. Soc. bras. Med. trop., 18: 47.56, 1985.

14.- LlLANOS CUENTAS, A.; ARANA, M.; MONTOYA, Y.; CAMPOS, M. \& ROMERO, G. - Aislamiento primario de Leishmania sp de pacientes en áreas endémicas del Peri. In: REUNIAO ANUAL SOBRE PESQUISA BA. SICA EM DOENÇA DE CHAGAS, 11., Caxambu, 1984 Programa e resumos. Ribeirăo Preto, PIDE: CNPq 1984. p. 58.

15. MAYRINK, W.; WILLIAMS, P.; COELHO, M. V.; DIAS, M.; VIANNA MARTINS, A.; MAGALHÃES, P. A.; DA COSTA, C. A.; FALCAO, A. R.; MELO, M. N. \& FALCAO, A. L. - Epidemiology of dermal leishmaniasis in the Rio Doce Valley State of Minas Gerais. Brazil, Ann. trop. Med. Parasit., 73: 124-137, 1979.

16. NETTO, E. M.; CUBA CUBA, C. A.; MARSDEN, P D. \& BARRETo, A. C. - El método de cultivo "in vitro" como instrumento práctico en el điagnóstico y ef aislamionto primario de Letshmanis brazikiensis braziliensis 1. Observaciones de Laboratório. Rev. Soc. bras. Med. trop., 1985 (apresentado para publicación)

17. PETERS, W.; EVANS, D. A. \& LANHAM, S. M. The importance of parisite identification in cases of Leishmaniasis. I, ray. Soc. Med., 36: 540-542, 1983. 
CUBA CUBA, C. A.; NETTO, E. M.; COSTA, J. L. M.; BARRETO, A. C. \& MARSDEN, P. D. - El cultivo "in vitro" como instrumento práctico para el diagnóstico y aislamiento primario de Leishmania braziliensis braviliensis. 2, Estudios en pacientes de áreas endémicas. Rev. Inst. Med. trop. São Paulo, 28:317.324, 1986.

18. SAR IIVA, N. G.; HOLGUIN, A. F.; Me MAHON PRATT, D. \& D'ALESSANDRO, A. - Mucocutaneous Leishmaniasis in Colombia: Leishmania braziliensis subspecies diversity. Amer. J. trop. Med. Hyg., 34: 714.720, 1985.

19. SHAW, J. J. \& LAINSON, R. - The "in vitro" cultivation of members of the Leishmanta braziliensis complex. Trans. roy. Soc. trop. Med. Hyg., 75: 127, 1981.

20 URJEL, R.; RECACOECHEA, M; LA FUENTE, C. \& ORELLANA A., H. - Simple method for the collection of material from cutaneous and mucocutaneous leish. maniasis Jesions. Trans. roy. Soc. trop. Med. Hyg, 77: 882-883, 2973.
21. WALTON, B. C.; SHAW, J. J. \& LAINSON, R. Observations on the "in vitro" cultivation of Leishmania braziliensis. J. Parasit., 63: 1118-1119, 1977.

22. WORLD FEALTH ORGANIZATION - Report of the Fourth Meeting of the Scientific Working Group on Immunology of Leishmgriasis. INDR/WORLD/BANK' WHO TDR (4), 1982.

23. WIRTH, D. F. \& Mc MAHON PRATT, D. - Rapid identification of Lefshmania species by specific hy. bridisation of kinetoplast DNA in cutaneous lesions Proc. nat. Acad. Sci. (Wash.), 79: 6999-7003, 1982.

Recebiđo para publicaçăo em 12/11/1985. 